\title{
Forecasting Yield of Major Crops in Different District of Middle Gujarat and North Gujarat Using Statistical Techniques
}

\author{
S.B. Yadav $^{1}{ }^{*}$, M.J. Vasani ${ }^{1}$, N.J. Chaudhari ${ }^{1}$ and Mayur Shitap ${ }^{2}$ \\ ${ }^{1}$ Department of Agricultural Meteorology, Anand Agricultural University, Anand - 388110 , \\ Gujarat, India \\ ${ }^{2}$ Department of Agricultural Statistics, Junagadh Agricultural University, \\ Junagadh-362001, India
}

*Corresponding author

\section{A B S T R A C T}

\begin{tabular}{|l|}
\hline Ke y w o r d s \\
$\begin{array}{l}\text { Regression model, } \\
\text { Weighted and unweighed } \\
\text { coefficient, Yield forecast } \\
\text { model, Kharif and rabi } \\
\text { crop }\end{array}$ \\
\hline Article Info \\
\hline $\begin{array}{l}\text { Accepted: } \\
\text { 15 October } 2018 \\
\text { Available Online: } \\
10 \text { November } 2018\end{array}$ \\
\hline
\end{tabular}

\section{Introduction}

India has a comprehensive system for collection of Agricultural Statistics.

Most of the Indian States have an official agency (Revenue administration) which collects and compiles crop area and production estimates at the village level and transmits it for aggregation at higher levels (national/state/district). These estimates suffer
Pre harvest forecast of agricultural production is essentially required for food security point of view. In this paper attempt has been made to develop model for forecasting the yield of kharif (Groundnut, paddy, maize) and rabi (wheat and mustard) crop of different district of middle Gujarat and north Gujarat using regression technique. The model were developed base on 30 years (1985 to 2015) district wise crop yield and weekly meteorological data and validated with 2 years (2010-11 \& 2011-12) and forecast were issued for 2012-13. The result showed that for kharif crop the model developed could explain 40 to $90 \%$ variation in groundnut yield 47 to $93 \%$ variation in paddy yield 54 to $87 \%$ variation in maize yield in different district of middle and north Gujarat. For rabi crops (wheat and mustard) the models explained about 60 to $90 \%$ variation in the yield in different districts. The $\mathrm{R}^{2}$ obtained were found to be significant at $\mathrm{P}=0.01$. During validation period $(2010 \& 11)$ the predicted yield deviations less than $10 \%$ of the reported yields were crop and districts. This revealed that the models can successfully be used for yield forecasting. The district wise yield forecast was issued for different crops for year 2012-13. The details of the findings are discussed in the paper. 
models were developed to study crop weather relationship but later they were applied to forecast (Jain, 1980; Agrawal, 1986).

Yield forecast models were developed for wheat and rice using weather variables and agricultural inputs on agro-climatic zone basis by (Agrawal et al., 2001). By coupling technology trend with weather variables, models were found to perform better Mallick et al., 2007). The present study provides yield forecast models for major crops for different district of middle and north Gujarat using technique developed at IASRI, New Delhi (Agrawal et al., 1980 \& Jain et al., 1980)

\section{Materials and Methods}

\section{Data used}

Twenty seven years (1985 to 2012) was collected for Directorate of Agriculture, Gandhinagar and corresponding weather data were collected from the agro-meteorological observatories situated in respective districts (Table 1). Crops and districts of middle and north Gujarat were selected based on terms of area and production of each crop districts for their significant contribution at state level. It may be seen that some station have little less no. of years of weather data. In Kharif season paddy, maize and groundnut were chosen while for rabi season wheat and mustard was chosen for yield prediction. In all the case data upto 2009 were used for development for model and two year (2010-11 and 2011-12) were used for validation purpose.

\section{Techniques applied for development of} district wise statistical models

Following the methodology suggested by Indian Agricultural Statistical Research Institute (IASRI), New Delhi (Agrawal et al., 1980; Jain et al., 1980).

The crop yield forecasting models for major growing districts were developed using stepwise regression analysis. Weather variables are used as independent variables which are related to crop responses such as yield and to account for the technological changes some function of time is used as independent variables. IASRI modified the model of Hendricks and Scholl (1943) by expressing the effects of changes in weather variables on yield as function of respective correlation coefficients between yield and weather variables (Table 2). This explains the relationship in a better way as it gives appropriate weightage to different periods. Under this assumption, the models were developed for studying the effects of weather variables on yield. These models are found to be better than the one suggested by Hendricks and Scholl in 1943 (Agrawal, et al., 1986; Mehta et al., 2000). The forecast model finally recommended is as follows

$Y=A_{0}+\sum_{i=1}^{p} \sum_{j=0}^{1} a_{i j} z_{i j}+\sum_{i \neq i=1}^{p} \sum_{j=0}^{1} a_{i i^{r} j} z_{i i^{r_{j}}}+c T+e$

Where,

$\mathrm{Z}_{\mathrm{ij}}=\sum_{w=1}^{m} r_{i w}^{j} x_{i w}$ and $\mathrm{Z}_{\mathrm{ii} j}=\sum_{w=1}^{m} r_{i i w}^{j} x_{i w} x_{i w}$

$r_{i w}$ - is correlation coefficient of yield with i-th weather variable in w-th period

$\mathrm{r}_{\mathrm{ii}}{ }^{\mathrm{w}}$ - is correlation coefficient (adjusted for trend effect) of yield with product of $i$-th and i'-th weather variables in $\mathrm{w}$-th period

$\mathrm{m}$ - is period of forecast

$\mathrm{P}$ - is number of weather variables used

e - is random error distributed as $\mathrm{N}(0, \sigma 2)$

The following eight weather variables were selected for development of models

Bright sunshine hour (BSS) 
Maximum temperature (Tmax)

Minimum temperature (Tmin)

Morning relative humidity (RH1)

Afternoon relative humidity ( $\mathrm{RH} 2)$

Morning vapour pressure (VP1)

Afternoon vapour pressure (VP2)

Rainfall (Rain)

For each weather variable, two weather indices were developed, one as simple accumulation of weather variable and the other one as weighted accumulation of weekly weather variable, weights being correlation coefficients of weather variable in respective weeks with yield (adjusted for trend effect, if present). Similarly, for interaction of weather variables, indices were generated using weekly products of weather variables taking two at a time. Stepwise regression technique was used to select the important weather indices. These weighted coefficients were finally regressed with the district yield to find out the final model. The final models were selected on the basis of highest $\mathrm{R}^{2}$ and the value of significance of $F$ test. Although for project work the models were separately developed for mid-season forecast at F1 stage (by $15^{\text {th }}$ September) and final forecast at F2 stage (by $15^{\text {th }}$ October) for kharif crops but here the models and results are presented for final and forecasts issued. Similarly for Rabi crops the models were developed using data upto to $20^{\text {th }}$ February

\section{Results and Discussion}

Development of district wise yield forecasting models for kharif 2012 using data up to $15^{\text {th }}$ October

The second $\left(\mathrm{F}_{2}\right)$ stage yield forecasting models were developed using the crop yield and weather data from $15^{\text {th }}$ June to $15^{\text {th }}$ October for kharif crops like paddy, groundnut and maize crops for the districts of Gujarat state (Table 3). The crop wise details are described as below.

\section{Paddy}

For Paddy crop for Anand the variables weighted Tmax $\left(Z_{21}\right)$, Time, and weighted Tmax*rainfall $\left(Z_{281}\right)$ found to be significant. Similarly the variables found significant for Baroda district were Time, weighted RH1 $\left(Z_{41}\right)$, weighted Tmin*RH2 $\left(Z_{251}\right)$ and for Panchmahal district the variables found significant were Time, weighted Tmax $\left(Z_{21}\right)$, weighted Tmax*rainfall $\left(\mathrm{Z}_{31}\right)$ and weighted Tmin $\left(Z_{31}\right)$. For Kheda district the variables that affected the yield significantly were Time, weighted $\mathrm{RH} 2 *$ rainfall $\left(\mathrm{Z}_{581}\right)$ and weighted Tmax*RH1 $\left(\mathrm{Z}_{241}\right)$. For Banaskantha district weighted Tmax*rainfall $\left(Z_{281}\right)$, Time and weighted Tmin $\left(Z_{31}\right)$ were found to be significantly responsible for yield prediction. Similarly for Dahod district the variables Time, weighted Tmax*rainfall $\left(\mathrm{Z}_{281}\right)$ and weighted Tmax $\left(Z_{21}\right)$ and for Bhavnagar district the variables weighted Tmin*rainfall $\left(\mathrm{Z}_{381}\right)$ were found to be significant. Similar results were also found for pre-harvest forecast of sugarcane. (Krishna and Suresh. 2010)

\section{Groundnut}

For Groundnut crop for Anand and Kheda district the variable weighted Tmax*Tmin $\left(\mathrm{Z}_{231}\right)$ alone found to be significantly responsible for yield prediction. For Baroda district Time, weighted coefficient for Tmax*RH2 $\left(Z_{251}\right)$, weighted coefficient for RH1 $\left(Z_{41}\right)$ were found to be significant. For Panchmahal and Dahod district weighted Tmin*RH2 $\left(\mathrm{Z}_{351}\right)$ and weighted RH2 $\left(\mathrm{Z}_{51}\right)$ respectively contributed significantly for yield prediction. For Banaskantha district weighted Tmax $\left(Z_{21}\right)$, Time, weighted coefficient for Tmax*RH1 $\left(Z_{241}\right)$ found to be significant. For Bhavnagar district the variables Time, weighted coefficient for Tmax*Tmin $\left(Z_{231}\right)$ and weighted $\mathrm{RH} 1 *$ rainfall $\left(\mathrm{Z}_{481}\right)$ were included in the model. Ramakrishna et al., 
(2003) have also developed forecast equations base on regression model, for total Indian food grain production using monsoon rainfall and soil index.

\section{Maize}

The maize yield forecasting models also performed well for the districts. For Anand districts the variables coefficient for Tmax*Tmin $\left(Z_{231}\right), \quad$ Time, weighted $\mathrm{RH} 2 *$ rainfall $\left(\mathrm{Z}_{581}\right)$, for Baroda district the variables weighted Tmin*RH2 $\left(\mathrm{Z}_{31}\right)$ and unweighted coefficient for RH1*RH2 $\left(Z_{31}\right)$ were found to be significant.

The Variables weighted RH1 $\left(Z_{31}\right)$ found to be significantly influencing the yield prediction for Panchmahal and Dahod district. Similarly the variables weighted Tmax*RH1 $\left(Z_{31}\right)$, weighted $\mathrm{RH} 2 *$ rainfall $\left(\mathrm{Z}_{31}\right)$ for Kheda district, the variables Time, weighted
Tmax*RH1 $\left(\mathrm{Z}_{31}\right)$ and weighted rainfall for Banaskantha district found to be significantly affecting the yield prediction. Similar study war carried out by Baweja, P.K. (2002).Predicted grain yield of maize on the basis of canopy temperature indices.

Development of district wise yield forecasting models for rabi 2012-13 using data up to $20^{\text {th }}$ February

\section{Wheat}

The crop yield forecasting models were developed during the rabi season 2012-13 using the weather data up to $20^{\text {th }}$ February for major growing district of crops viz., Wheat (Kheda, Anand, Bhavnagar, Panchmahal, Dahod, Banaskantha, Sabarkantha and Baroda), Mustard (Banaskantha and Sabarkantha) (Table 4).

Table.1 Data Used for the Analysis

\begin{tabular}{|c|c|c|c|c|}
\hline \multirow[t]{2}{*}{ Districts } & \multirow{2}{*}{$\begin{array}{l}\text { Agro-met } \\
\text { stations }\end{array}$} & \multirow{2}{*}{$\begin{array}{c}\text { Period } \\
\text { available } \\
\text { weather data }\end{array}$} & \multicolumn{2}{|c|}{ Crops selected } \\
\hline & & & Kharif & Rabi \\
\hline Anand & Anand & $1985-2012$ & Paddy, maize \& groundnut & Wheat \\
\hline Kheda & Nawagam & $1985-2012$ & Paddy, maize \& groundnut & Wheat \\
\hline Banaskantha & S K Nagar & $1985-2012$ & Paddy, maize \& groundnut & $\begin{array}{l}\text { Wheat \& } \\
\text { mustard }\end{array}$ \\
\hline Panchmahal & Godhra & $1989-2012$ & Paddy, maize \& groundnut & Wheat \\
\hline Baroda & Baroda & $1992-2012$ & Paddy, maize \& groundnut & Wheat \\
\hline Bhavnagar & Mahuva & $1987-2012$ & Paddy \& groundnut & Wheat \\
\hline Dahod & Dahod & $1989-2012$ & Paddy, maize \& groundnut & Wheat \\
\hline Sabarkantha & Khedbrahma & $1985-2012$ & Paddy, maize \& groundnut & $\begin{array}{l}\text { Wheat \& } \\
\text { mustard }\end{array}$ \\
\hline
\end{tabular}


Table.2 Variables used in model development and their description

\begin{tabular}{|c|c|c|c|}
\hline Symbols & Description & Symbols & Description \\
\hline $\mathrm{Z10}$ & Unweighted coefficients for BSS & Z11 & Weighted coefficients for BSS \\
\hline $\mathbf{Z 2 0}$ & Unweighted coefficients for Tmax & $\mathrm{Z} 21$ & Weighted coefficients for Tmax \\
\hline $\mathbf{Z 3 0}$ & Unweighted coefficients for Tmin & Z31 & Weighted coefficients for Tmin \\
\hline $\mathbf{Z 4 0}$ & Unweighted coefficients for morning hours humidity & $\mathrm{Z} 41$ & Weighted coefficients for morning hours humidity \\
\hline $\mathbf{Z 5 0}$ & Unweighted coefficients for evening hours humidity & $\mathrm{Z} 51$ & Weighted coefficients for evening hours humidity \\
\hline $\mathrm{Z60}$ & $\begin{array}{l}\text { Unweighted coefficients for Morning hours Vapour } \\
\text { pressure }\end{array}$ & Z61 & $\begin{array}{l}\text { Weighted coefficients for Morning hours Vapour } \\
\text { pressure }\end{array}$ \\
\hline $\mathbf{Z 7 0}$ & $\begin{array}{l}\text { Unweighted coefficients for Evening hours Vapour } \\
\text { pressure }\end{array}$ & $\mathrm{Z71}$ & $\begin{array}{l}\text { Weighted coefficients for Evening hours Vapour } \\
\text { pressure }\end{array}$ \\
\hline $\mathbf{Z 8 0}$ & Unweighted coefficients for Rainfall & $\mathrm{Z} 81$ & Weighted coefficients for Rainfall \\
\hline $\mathrm{Z} 120$ & Unweighted coefficients for BSS*Tmax & $\mathrm{Z} 121$ & Weighted coefficients for BSS*Tmax \\
\hline Z 130 & Unweighted coefficients for BSS*Tmin & Z 131 & Weighted coefficients for BSS*Tmin \\
\hline Z 140 & $\begin{array}{l}\text { Unweighted coefficients for BSS*morning hours } \\
\text { humidity }\end{array}$ & Z 141 & $\begin{array}{l}\text { Weighted coefficients for BSS*morning hours } \\
\text { humidity }\end{array}$ \\
\hline $\mathrm{Z} 150$ & $\begin{array}{l}\text { Unweighted coefficients for BSS* Evening hours } \\
\text { Humidity }\end{array}$ & Z 151 & $\begin{array}{l}\text { Weighted coefficients for BSS*morning hours } \\
\text { humidity }\end{array}$ \\
\hline $\mathrm{Z} 160$ & $\begin{array}{l}\text { Unweighted coefficients for BSS*Morning hours } \\
\text { Vapour Pressure }\end{array}$ & Z 161 & $\begin{array}{l}\text { Weighted coefficients for BSS*Morning hours } \\
\text { Vapour Pressure }\end{array}$ \\
\hline Z 170 & $\begin{array}{l}\text { Unweighted coefficients for BSS*Evening hours } \\
\text { Vapour Pressure }\end{array}$ & Z 171 & $\begin{array}{l}\text { Weighted coefficients for BSS*Evening hours } \\
\text { Vapour Pressure }\end{array}$ \\
\hline $\mathbf{Z} 230$ & Unweighted coefficients for Tmax*Tmin & Z 231 & Weighted coefficients for Tmax*Tmin \\
\hline Z240 & $\begin{array}{l}\text { Unweighted coefficients for Tmax* morning hours } \\
\text { humidity }\end{array}$ & Z241 & $\begin{array}{l}\text { Weighted coefficients for Tmax* morning hours } \\
\text { humidity }\end{array}$ \\
\hline $\mathbf{Z 2 5 0}$ & $\begin{array}{l}\text { Unweighted coefficients for Tmax* Evening hours } \\
\text { Humidity }\end{array}$ & Z251 & $\begin{array}{l}\text { Weighted coefficients for Tmax* Evening hours } \\
\text { Humidity }\end{array}$ \\
\hline $\mathbf{Z 2 6 0}$ & $\begin{array}{l}\text { Unweighted coefficients for Tmax*Morning hours } \\
\text { Vapour Pressure }\end{array}$ & Z261 & $\begin{array}{l}\text { Weighted coefficients for Tmax*Morning hours } \\
\text { Vapour Pressure }\end{array}$ \\
\hline $\mathbf{Z 2 7 0}$ & $\begin{array}{l}\text { Unweighted coefficients for Tmax*Evening hours } \\
\text { Vapour Pressure }\end{array}$ & Z271 & $\begin{array}{l}\text { Weighted coefficients for Tmax*Evening hours } \\
\text { Vapour Pressure }\end{array}$ \\
\hline $\mathbf{Z 2 8 0}$ & Unweighted coefficients for Tmax*Rainfall & Z280 & Weighted coefficients for Tmax*Rainfall \\
\hline Z 340 & $\begin{array}{l}\text { Unweighted coefficients for Tmin*Morning hours } \\
\text { Humidity }\end{array}$ & Z 341 & $\begin{array}{l}\text { Weighted coefficients for Tmin* Morning hours } \\
\text { Humidity }\end{array}$ \\
\hline Z 350 & $\begin{array}{l}\text { Unweighted coefficients for Tmin*Evening hours } \\
\text { Humidity }\end{array}$ & Z 351 & $\begin{array}{l}\text { Weighted coefficients for Tmin* Evening hours } \\
\text { Humidity }\end{array}$ \\
\hline $\mathrm{Z} 360$ & $\begin{array}{l}\text { Unweighted coefficients for Tmin*Morning hours } \\
\text { Vapour Pressure }\end{array}$ & Z 361 & $\begin{array}{l}\text { Weighted coefficients for Tmin*Morning hours } \\
\text { Vapour Pressure }\end{array}$ \\
\hline Z 370 & $\begin{array}{l}\text { Unweighted coefficients for Tmin*Evening hours } \\
\text { Vapour Pressure }\end{array}$ & $\mathrm{Z} 371$ & $\begin{array}{l}\text { Weighted coefficients for Tmin*Evening hours } \\
\text { Vapour Pressure }\end{array}$ \\
\hline Z 380 & Unweighted coefficients for Tmin*Rainfall & $\mathrm{Z} 381$ & Weighted coefficients for Tmin*Rainfall \\
\hline $\mathrm{Z} 450$ & $\begin{array}{l}\text { Unweighted coefficients for Morning hours Humidity } \\
\text { *Evening hours Humidity }\end{array}$ & $\mathrm{Z} 451$ & $\begin{array}{l}\text { Weighted coefficients for Morning hours } \\
\text { Humidity *Evening hours Humidity }\end{array}$ \\
\hline $\mathrm{Z} 460$ & $\begin{array}{l}\text { Unweighted coefficients for Morning hours Humidity } \\
\text { *Morning hours Vapour Pressure }\end{array}$ & Z461 & $\begin{array}{l}\text { Weighted coefficients for Morning hours } \\
\text { Humidity *Morning hours Vapour Pressure }\end{array}$ \\
\hline Z470 & $\begin{array}{l}\text { Unweighted coefficients for Morning hours Humidity } \\
\text { *Evening hours Vapour Pressure }\end{array}$ & Z471 & $\begin{array}{l}\text { Weighted coefficients for Morning hours } \\
\text { Humidity *Evening hours Vapour Pressure }\end{array}$ \\
\hline $\mathbf{Z 4 8 0}$ & $\begin{array}{l}\text { Unweighted coefficients for Morning hours Humidity } \\
\text { *Rainfall }\end{array}$ & Z481 & $\begin{array}{l}\text { Weighted coefficients for Morning hours } \\
\text { Humidity *Rainfall }\end{array}$ \\
\hline Z 560 & $\begin{array}{l}\text { Unweighted coefficients for Evening hours Humidity } \\
\text { *Morning hours Vapour Pressure }\end{array}$ & Z 561 & $\begin{array}{l}\text { Weighted coefficients for Evening hours Humidity } \\
\text { *Morning hours Vapour Pressure }\end{array}$ \\
\hline Z 570 & $\begin{array}{l}\text { Unweighted coefficients for Evening hours Humidity } \\
\text { *Evening hours Vapour Pressure }\end{array}$ & Z 571 & $\begin{array}{l}\text { Weighted coefficients for Evening hours Humidity } \\
\text { *Evening hours Vapour Pressure }\end{array}$ \\
\hline Z 580 & $\begin{array}{l}\text { Unweighted coefficients for Evening hours Humidity } \\
\text { *Rainfall }\end{array}$ & Z 581 & $\begin{array}{l}\text { Weighted coefficients for Evening hours Humidity } \\
\text { *Rainfall }\end{array}$ \\
\hline Z670 & $\begin{array}{l}\text { Unweighted coefficients for Morning hours } \\
\text { Vapour Pressure* Evening hours Vapour Pressure }\end{array}$ & Z 671 & $\begin{array}{l}\text { Weighted coefficients for Morning hours Vapour } \\
\text { Pressure*Evening hours Vapour Pressure }\end{array}$ \\
\hline
\end{tabular}


Table.3 District wise crop yield forecasting model using data up to 15 October (F2)

\begin{tabular}{|c|c|c|c|c|}
\hline Crop & District & Regression equation & $\mathbf{R}^{2}$ & $\begin{array}{c}\text { Forecast yield } \\
(\mathrm{Kg} / \mathrm{ha}) \text { for } \\
2012\end{array}$ \\
\hline \multirow[t]{7}{*}{ Groundnut } & Anand & $\mathrm{Y}=4520.03+3.47 * \mathrm{Z} 231$ & 0.53 & 1985 \\
\hline & Baroda & $\mathrm{Y}=-697.03+47.02 * \mathrm{TIME}+0.19 * \mathrm{Z} 251+6.77 * \mathrm{Z} 41$ & 0.93 & 1960 \\
\hline & Panchmahal & $\mathrm{Y}=1479.39+0.42 * \mathrm{Z} 351$ & 0.40 & 1442 \\
\hline & Kheda & $\mathrm{Y}=3258.66+3.16 * \mathrm{Z} 231$ & 0.59 & 1598 \\
\hline & Banaskantha & $\begin{array}{l}\mathrm{Y}=2347.30+63.26 * \mathrm{Z} 21+18.04 * \mathrm{TIME}+ \\
0.68 * \mathrm{Z} 241\end{array}$ & 0.68 & 1548 \\
\hline & Dahod & $\mathrm{Y}=1498.48+11.36 * \mathrm{Z} 51$ & 0.55 & 1564 \\
\hline & Bhavnagar & $\begin{array}{l}\mathrm{Y}=1063.11+29.63 * \mathrm{TIME}+0.95 * \mathrm{Z} 231+ \\
0.03 * \mathrm{Z} 481\end{array}$ & 0.74 & 1272 \\
\hline \multirow[t]{7}{*}{ Paddy } & Anand & $\begin{array}{l}\mathrm{Y}=4268.57+37.88 * \mathrm{Z} 21+32.63 * \mathrm{TIME}+ \\
0.15 * \mathrm{Z} 281\end{array}$ & 0.73 & 2453 \\
\hline & Baroda & $\mathrm{Y}=2119.14+50.86 * \mathrm{TIME}+8.85 * \mathrm{Z} 41+0.17 * \mathrm{Z} 351$ & 0.96 & 1751 \\
\hline & Panchmahal & $\begin{array}{l}\mathrm{Y}=1107.62+61.56 * \mathrm{TIME}+53.61 * \mathrm{Z} 21+ \\
0.05 * \mathrm{Z} 281+15.41 * \mathrm{Z} 31\end{array}$ & 0.97 & 2159 \\
\hline & Kheda & $\mathrm{Y}=315.59+37.42 * \mathrm{TIME}+0.04 * \mathrm{Z} 581+0.37 * \mathrm{Z} 241$ & 0.79 & 2231 \\
\hline & Banaskantha & $\mathrm{Y}=112.59+0.05 * \mathrm{Z} 281-10.39 * \mathrm{TIME}+43.53 * \mathrm{Z} 31$ & 0.70 & 1136 \\
\hline & Dahod & $\begin{array}{l}\mathrm{Y}=2383.66+51.88 * \mathrm{TIME}+0.10 * \mathrm{Z} 281+ \\
36.33 * \mathrm{Z} 21\end{array}$ & 0.84 & 1775 \\
\hline & Bhavnagar & $\mathrm{Y}=1641.57+0.20 * \mathrm{Z} 381$ & 0.47 & 1651 \\
\hline \multirow[t]{6}{*}{ Maize } & Anand & $\begin{array}{l}Y=-166.71+1.98 * Z 231+38.16 * \text { TIME }+ \\
0.06 * Z 581\end{array}$ & 0.67 & 2507 \\
\hline & Baroda & $\mathrm{Y}=1744.56+0.36 * \mathrm{Z} 351-0.01 * \mathrm{Z} 450$ & 0.85 & 1673 \\
\hline & Panchmahal & $\mathrm{Y}=723.74+24.43 * \mathrm{Z} 41$ & 0.61 & 1392 \\
\hline & Kheda & $\mathrm{Y}=-2352.12+0.47 * \mathrm{Z} 241+0.04 * \mathrm{Z} 581$ & 0.54 & 1668 \\
\hline & Banaskantha & $\mathrm{Y}=5724.22+55.87 * \mathrm{TIME}+0.42 * \mathrm{Z} 241+2.50 * \mathrm{Z} 81$ & 0.82 & 2394 \\
\hline & Dahod & $\mathrm{Y}=1567.35+22.71 * \mathrm{Z} 41$ & 0.59 & 1447 \\
\hline
\end{tabular}

Table.4 Development of district wise yield forecasting for rabi 2012-13

\begin{tabular}{|c|c|c|c|c|}
\hline Crop & Districts & Regression equation & $\mathbf{R}^{2}$ & $\begin{array}{l}\text { Forecasted yield } \\
\text { (Kg/ha) for } \\
2012-13\end{array}$ \\
\hline \multirow[t]{8}{*}{ Wheat } & Anand & $\mathrm{Y}=1190.56+53.37 *$ Time $+1.12 * \mathrm{Z} 351$ & 0.89 & 2841 \\
\hline & Baroda & $\mathrm{Y}=6294.75+134.26 * \mathrm{Z} 11+0.33 * \mathrm{Z} 241+0.11 * \mathrm{Z} 140$ & 0.89 & 2602 \\
\hline & Panchmahal & $\begin{array}{l}\mathrm{Y}=1345.78+1.48 * \mathrm{Z} 141+40.76 * \mathrm{Z} 21+3.94 * \mathrm{Z} 20- \\
0.54 * \mathrm{Z} 160\end{array}$ & 0.89 & 2020 \\
\hline & Kheda & $\begin{array}{l}Y=1193.44+60.29 * \text { Time }+39.30 * Z 61+0.69 * Z 151 \\
+1.13 * Z 141\end{array}$ & 0.94 & 3052 \\
\hline & Banaskantha & $\mathrm{Y}=3729.64+13.31 * \mathrm{Z} 161+1.07 * \mathrm{Z} 231$ & 0.60 & 2641 \\
\hline & Dahod & $\mathrm{Y}=3105.66+236.79 * \mathrm{Z} 11+0.31 * \mathrm{Z} 251+0.44 * \mathrm{Z} 341$ & 0.85 & 2054 \\
\hline & Bhavnagar & $\mathrm{Y}=-972.03+52.15 *$ Time $+378.02 * \mathrm{Z} 11+0.81 * \mathrm{Z} 241$ & 0.85 & 3016 \\
\hline & Sabarkantha & $\mathrm{Y}=5707.92+38.36 * \mathrm{Z} 21+3.10 * \mathrm{Z} 171+0.54 * \mathrm{Z} 141$ & 0.73 & 2474 \\
\hline \multirow[t]{2}{*}{ Mustard } & Banaskantha & $\mathrm{Y}=3581.70+0.95 * \mathrm{Z} 141+85.66 * \mathrm{Z} 21$ & 0.67 & 1569 \\
\hline & Sabarkantha & $\mathrm{Y}=1495.87+13.93 *$ Time $+0.39 * \mathrm{Z} 341+1.84 * \mathrm{Z} 121$ & 0.84 & 1430 \\
\hline
\end{tabular}


Table.5 Validation of statistical model in 2010 and 2011 for groundnut, paddy, maize and wheat different districts of Gujarat

\begin{tabular}{|l|c|c|c|c|c|c|c|c|}
\hline Districts & \multicolumn{3}{|l|}{$\begin{array}{l}\text { Percent deviation of forecasts from } \\
\text { observed yield (2010) }\end{array}$} & \multicolumn{3}{l}{$\begin{array}{l}\text { Percent deviation of forecasts from } \\
\text { observed yield (2011) }\end{array}$} \\
\hline Anand & Groundnut & Maize & Paddy & Wheat & Groundnut & Maize & Paddy & wheat \\
\hline Bananskantha & 7.1 & -7.9 & 2.1 & 3.2 & -2.9 & -5.0 & 0.3 & -4.5 \\
\hline Bhavnagar & 4.9 & - & -9.0 & -4.0 & 4.0 & - & 8.7 & -1.9 \\
\hline Dahod & 0.8 & 6.7 & 2.4 & -5.5 & 4.1 & -6.9 & 8.5 & 3.0 \\
\hline Kheda & 8.0 & 8.0 & -5.6 & 3.7 & -8.2 & 7.2 & -7.0 & 7.5 \\
\hline Panchmahal & 6.7 & 4.2 & -5.4 & -6.3 & 0.4 & 8.4 & 2.4 & -3.8 \\
\hline Vadodara & 3.6 & 8.4 & 7.3 & 1.3 & 2.3 & 4.8 & -1.6 & 6.7 \\
\hline
\end{tabular}

Fig.1 Comparison with estimated yield by statistical method, crop weather simulation model (DSSAT) and average yield of rice, maize and groundnut for the different districts of Gujarat

a AVERAGE YIELD — ESTIMATED YIELD (STATISTICAL METHOD) — ESTIMATED YIELD (DSSAT)

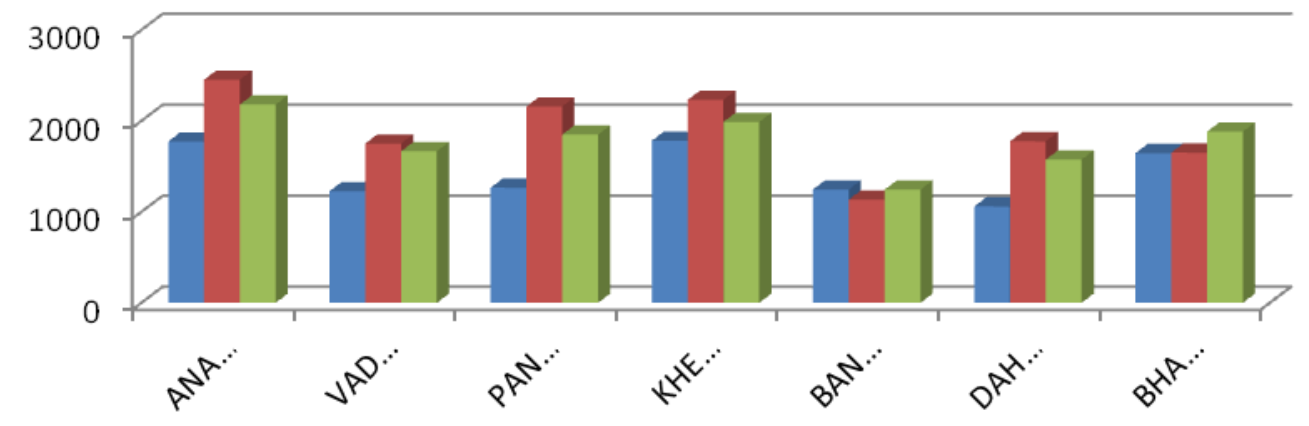

aVERAGE YIELD ESTIMATED YIELD (STATISTICAL METHOD) — ESTIMATED YIELD (DSSAT)
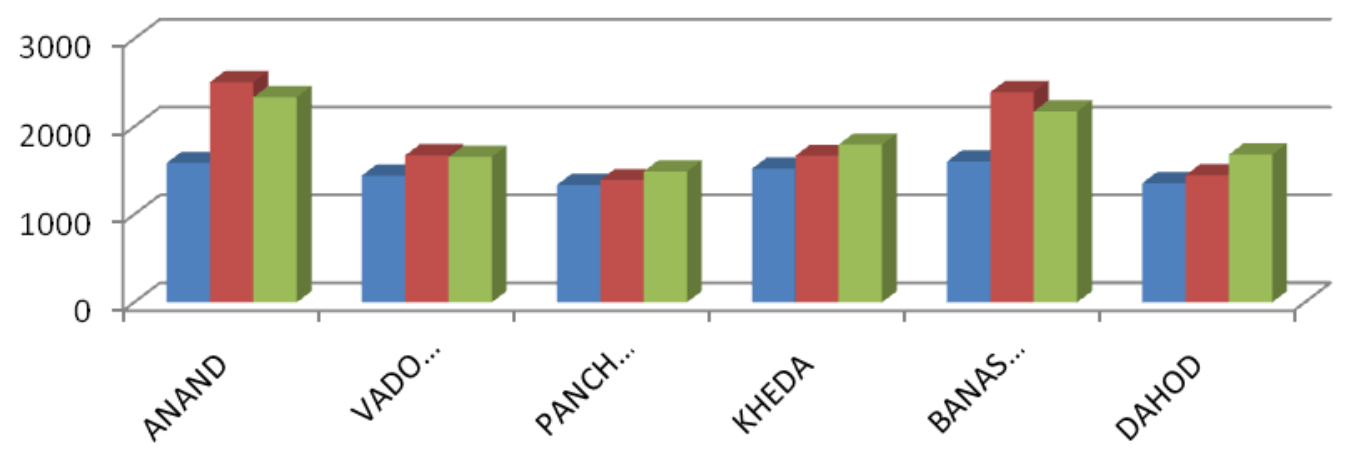


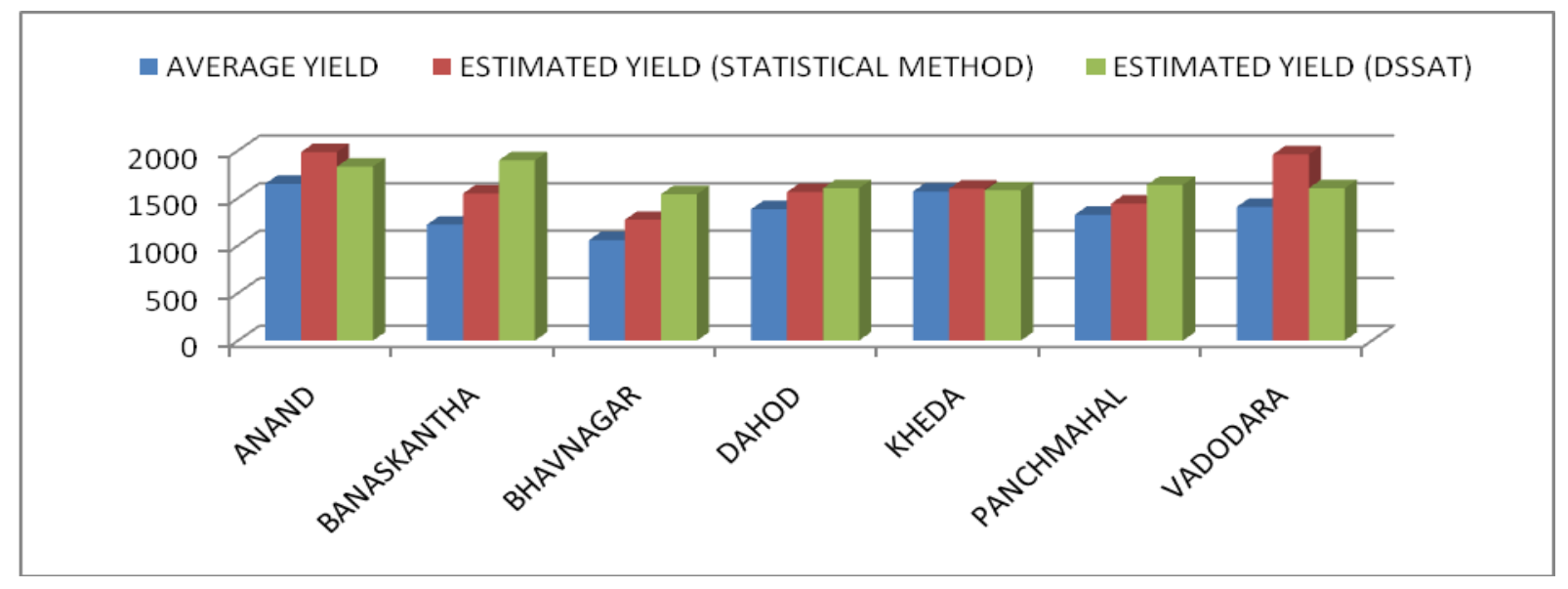

The wheat yield prediction models for wheat growing districts were developed. There was quite strong relationship was found between actual yield and weather variables. For Anand district variables weighted $\mathrm{BSS}\left(\mathrm{Z}_{511}\right)$, weighted Tmax*RH1 $\left(\mathrm{Z}_{241}\right)$ and unweighted BSS*RH1 $\left(Z_{741}\right)$ were found to be significant. The variables found significant for Panchmahal district were weighted BSS*RH1 $\left(Z_{141}\right)$, weighted and unweighted $\operatorname{Tmax}\left(\mathrm{Z}_{20}\right)$ and weighted BSS*VP1 $\left(Z_{160}\right)$. For Kheda district the variables Time, weighted VP1 $\left(Z_{61}\right)$, weighted $\mathrm{BSS} * \mathrm{RH} 2\left(\mathrm{Z}_{151}\right)$, weighted $\mathrm{BSS} * \mathrm{RH} 1$ $\left(Z_{141}\right)$ and for Banaskantha district weighted BSS*VP1 $\left(Z_{161}\right)$ and weighted Tmax*Tmin $\left(Z_{231}\right)$ were found to be significantly responsible for yield prediction. The variables weighted BSS $\left(Z_{11}\right)$, weighted Tmax*RH1 $\left(Z_{241}\right)$ for Bhavnagar district and for Sabarkantha district the variables weighted $T \max \left(Z_{21}\right)$, weighted BSS*VP2 $\left(Z_{171}\right)$ and weighted BSS*RH1 $\left(Z_{141}\right)$ were included in the yield prediction model. Similar types of results were obtained by Agrwal and Aditya (2012) for yield prediction of wheat.

\section{Mustard}

For Mustard crop the variables found significant for Banaskantha district were weighted BSS*RH1 $\left(\mathrm{Z}_{141}\right)$ and weighted Tmax $\left(\mathrm{Z}_{21}\right)$. For Sabarkantha district the Time, weighted Tmin*RH1 $\left(Z_{241}\right)$, weighted BSS*Tmax $\left(Z_{121}\right)$ were found to be significant for yield prediction.
Crop yield prediction using crop growth simulation model (DSSAT v4.5)

The crop yield prediction was made using the DSSAT ( $\mathrm{v}$ 4.5) crop growth simulation model (Table 4). The model was calibrated using the data (Crop and Weather) from 1990 to 2000 from all the stations and crops.

Data from 2001 to 2011 was used for validation of crop growth simulation model. The results presented in Figure 1 shows that there is no much difference forecasted crop yield by both the methods.

\section{Validation of model}

A comparison between the actual and predicted values of groundnut, paddy, maize and wheat yield for Anand, Baroda, Panchmahal, Kheda, Banaskantha, Dahod and Bhavnagar districts which were used in developing the forecast models, is presented in table 5. The results show that the percentage of deviations from the actual yield and forecasted yield is acceptable range raged between \pm 0.3 to 9.0 percent.

The results obtained by both the methods were nearly gave the same results. So from the results it can be concluded that both the methods are useful for the yield prediction purpose.

The DSSAT 4.5 crop growth simulation model gives the prediction considering the weather, 
soil and crop management data while statistical models are based on weather parameters only.

So if one is having all the data set (weather, soil and crop management data) it is better to go for crop growth simulation model otherwise statistical models also can serve the purpose if soil and crop management data are not available.

Using the forecast model, pre-harvest estimates of different crop yield for different districts of Gujarat could be computed successfully very much in advance before the actual harvest.

As the data used for developing this model is of high degree of accuracy, its reliability is also high. Further, this model will produce more accurate results depending on the accuracy of input data provided.

\section{References}

Agrawal Ranjana and Aditya, K. (2012). Use of discrimination function analysis for forecasting of crop yield. Mausam, 63(3):455-458

Agrawal, R., Jain, R.C. and Mehta, S.C. (2001). Yield forecast based on weather variables and agricultural inputs on agro climatic zone basis. Ind. J. Agri. Sci.

Agrawal, R., Jain, R.C., Jha, M.P. (1986). Models for studying rice crop-weather relationship, Mausam 37(1), 67-70.

Agrawal, R., Jain, R.C., Jha, M.P. and Singh, D. (1980). Forecasting of rice yield using climatic basis of weather variables. $J$. Agromet. 6(2), 223-228.

Baweja, P.K. (2002). Predicting grain yield in maize: canopy temperature based regression indices. J. Agromet. 4 (2), 177179.

Hendrick, W.A. and Scholl. J.E. (1943). Technique in measuring joint relationship the joint effects of temperature and precipitation on crop yield. North Carolina Agric. Exp.Sta. Tech.Bull., 74.

Jain, R.C., Agrawal, Ranjana and Jha, M.P. (1980). Effect of climatic variables on rice yield and its forecast. Mausam, 31(4), 591-596.

Krishna, S.R. and Suresh M.K. (2010). A study on pre-harvest forecast of sugarcane yield using climatic variables. Statistics and Applications.7\&8, Nos. 1\&2, 2009-10 (New Series), pp. 1-8

Mall, R.K. and Gupta, B.R.D. (2000).Wheat yield models based on meteorological parameters. J. Agromet. 2(1), 83-87.

Mallick, K., Mukherjee, J., Bal, S.K., Bhalla, S.S., Hundal, S.S. (2007). Real time rice yield forecasting over Central Punjab region using crop weather regression model. J. Agromet. 9(2), 158-166.

Mehta, S.C., Agrawal, R. and Singh V.P.N. (2000). Strategies for composite forecast. J. Ind. Soc. Ag. Stat., 53(3), 262-272.

Ramakrishna, Y.S., Singh H.P. and Maheshwara Rao (2003). Weather basis indices for forecasting national food grain production. J. Agrometeorol, 11 (2): 152155.

\section{How to cite this article:}

Yadav, S.B., M.J. Vasani, N.J. Chaudhari and Mayur Shitap. 2018. Forecasting Yield of Major Crops in Different District of Middle Gujarat and North Gujarat Using Statistical Techniques. Int.J.Curr.Microbiol.App.Sci. 7(11): 2202-2210. doi: https://doi.org/10.20546/ijcmas.2018.711.246 